\title{
Integrated Design for Fatigue Life Estimation of Structures
}

\author{
Radouane Akrache $1,{ }^{*}-$ Jian $\mathrm{Lu}^{2}$ \\ ${ }^{1}$ Laboratoire d'Ingénierie des Systèmes de Versailles, \\ Université de Versailles Saint-Quentin-en-Yvelines, France \\ 2Laboratoire des Systèmes Mécanique et Ingénierie Simultanée, \\ Université de Technologie de Troyes, France
}

The design of a product or a mechanical system involves several stages, starting with the development of product specifications and ending with its final destruction. The aim of our research is to develop appropriate tools by proposing new operating methodologies and new computer tools to link up material development process with the mechanical design. In the first part, we will outline the overall approach adopted, and describe an integrated design method for estimating the fatigue life of mechanical structures. In the second part, we will discuss prestressing processes and take them into account in our approach. The third part is devoted to developing a calculation code for determining the multiaxial fatigue life of $3 D$ structures.

(C)2011 Journal of Mechanical Engineering. All rights reserved.

Keywords: multiaxial fatigue life, integrated design, finite element method, fatigue criteria

\section{INTRODUCTION}

To achieve the aim of integrated design of mechanical systems, research into integration problems of and on a more fundamental level, the development of modelling tools and experimental control techniques are indispensable. Several factors have been integrated, corresponding to the optimization of mechanical systems, modelling of non-linear problems, experimental measurements, surface treatment, and the study of new materials and new manufacturing processes. In the field of optimization, a special resolution method has been developed [1]. It shows that, based on several concepts, a design problem can be expressed as a problem of optimization. This, in turn, allows us to produce a calculation code for non-linear optimization with mixed variables. These concepts can also be used to optimize the geometry of a part according to criteria such as cost, endurance and lifetime. In currently available software, a mechanical system is represented as the sum total of the volumes of its different components. This type of representation does not enable the user to easily design a mechanical system. A certain number of fundamental notions have also been left aside in current CAD systems. We therefore, propose adding several modelling and optimization modules to these tools. Another module i.e. the calculation of fatigue life is also integrated into this global approach as indicated in Fig. 1.

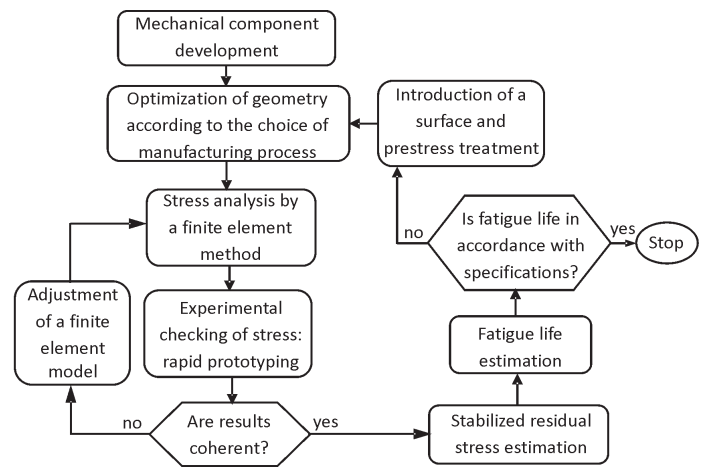

Fig. 1. Flow-chart of fatigue life evaluation during the design phase

\section{TAKING RESIDUAL STRESS INTO ACCOUNT}

\subsection{Definition}

Residual stress is generally defined as the stress that persists in a mechanical component which is not subjected to outside stress. Residual stress exists in practically all metal parts. It corresponds to the mechanical and metallurgic history of each point in the component and the component as a whole during the manufacturing 
process. It generally exists on three levels depending on the scale on which it is observed: (i) macroscopic stress; (ii) stress due to the heterogeneity and anisotropy of each crystal or grain in a mechanical polycrystalline, and (iii) microscopic stress. In structural analysis, macroscopic residual stress is used and modelled.

\subsection{Integration of Residual Stress into Fatigue Life Calculation}

The integration of residual stress into a computable calculation must be gradual and can be separated into several phases. Currently, little direct consideration is given to the residual stress parameter in design. In specifications, requirements that are often closely linked to residual stress without specially mentioning it, are made. In the first phase of integration, the notion of semiquantitativity can be used to evaluate the increase in performance in terms of lifetime or limit of endurance. Several manufacturing processes and surface treatments develop residual stress, which tends to increase the fatigue life and the limit of endurance. These processes include shot-peening, cold rolling and nitriding. The second phase consists in developing analytic and numerical calculation methods integrating residual stress. The method which takes residual stress into account in our approach is indicated in Fig. 2.

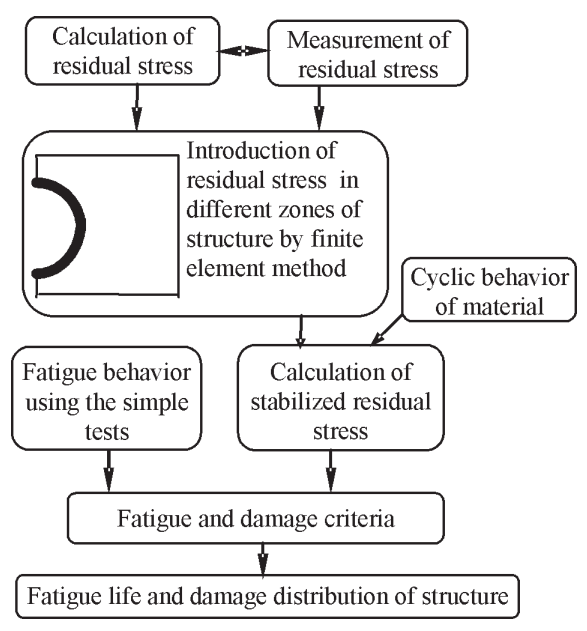

Fig. 2. Taking residual stress into account

On an experimental level, recent developments have enabled residual stress profiles induced by various industrial processes [2] to be obtained. It has been known for a long time that residual stress can be relaxed when components are subjected to cyclic loading. Using the 2D finite element method, we have modelled residual stress relaxation and the influence of the various factors involved. A criterion for determining the stability of the residual stress as a function of the cyclic characteristics of the materials [3] and [4] has been proposed. For a material with cyclic softening, there is gradual relaxation of residual stress whereas, for a material with cyclic hardening, the residual stress is stabilised after several fatigue cycles (Fig. 3).

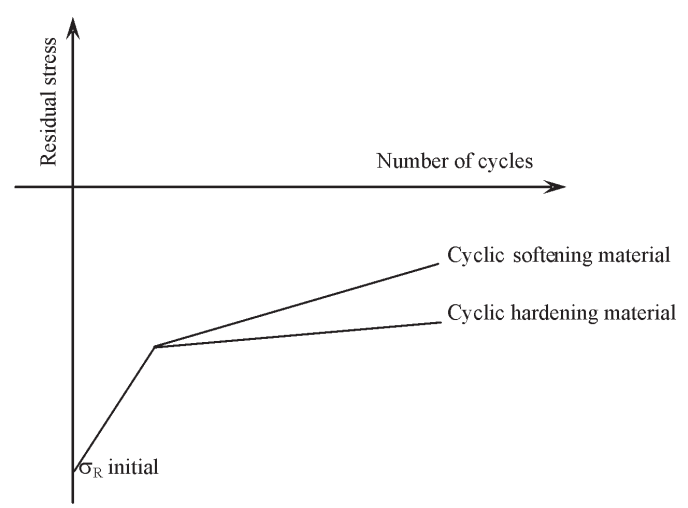

Fig. 3. Influence of cyclic properties on the fatigue resistance of materials

Continuation of this research consists from developing methods for calculating the relaxation of residual stress in three-dimensional structures under complex thermomechanical loading. The major difficulties lies in introducing residual stress profiles measured on 3D structures. Recent studies have improved the possibility of carrying out this type of calculation [5].

If the different effects of residual stress on the appearance of fatigue in structures is known, then a quantitative estimatation of the fatigue life can be given. By limiting the estimation of the lifetime of a component to the initial appearance of fatigue cracking, the problem of predicting the lifetime of a mechanical component subjected to a high number of cycles ( $>5.10^{4}$ cycles) can be analysed. To achieve this aim, a tool for predicting fatigue life must be developed. In the next section this calculation code that will integrate the residual stresses is presented. 


\section{DEVELOPMENT OF A FATIGUE LIFE CALCULATION CODE}

\subsection{Introduction}

Predicting the fatigue life of threedimensional structures is always a tricky problem, because it must assess the length of time during which man and machine are safe. Whenever new parts are developed in industry, their fatigue life has to be estimated to check the durability of the corresponding mechanism. To carry out this estimation, experiment-based criteria must be established.

\subsection{Fatigue Criteria}

The scientific literature includes several suggestions for fatigue criteria. Some were originally applied to particular loading conditions. In order to produce a fatigue design for structures under dynamic loading, the safety range limited by a threshold needs to be calculated. Above this threshold, the material will be damaged or cracks will appear. For high cycle fatigue, most of the criteria are stress-based formulae. In practice, the critical values for these parameters are calculated and checked that the loading case obtained is within the range of safety.

\subsubsection{Crossland and Sines Criteria}

The plasticity model used as a basis for these criteria is the Von Mises criterion which suggests that plasticity appears when the octahedral shear (shear relative to the facet leaning equally on the principal axes) reaches a certain limit. These two criteria are expressed by a linear relationship between the octahedral shear amplitude and the hydrostatic pressure (mean pressure during the cycle for the Sines criterion [6], maximum pressure for the Crossland criterion [7]). If fixed coordinate axes towards the material are chosen $(x, y, z)$ the stress variation tensor $\left(\Delta \Sigma_{i j}\right)$ between two instants, $t_{1}$ and $t_{2}$, of the cycles is written:

$$
\left[\Delta \Sigma_{i j}\left(t_{1}, t_{2}\right)\right]=\left[\Sigma_{i j}\left(t_{2}\right)\right]-\left[\Sigma_{i j}\left(t_{1}\right)\right] .
$$

The second invariant $J_{2}$ of this tensor is intrinsic: an intrinsic octahedral shear amplitude can be defined by:

$$
\Delta \tau_{o c t, a}=\frac{1}{2}\left[\frac{2}{3} \Delta J_{2}\right]^{\frac{1}{2}},
$$

where $\Delta J_{2}$ is the largest possible value of the second invariant, obtained by double maximisation:

$$
\Delta J_{2}=\max _{t_{1}}\left\{\max _{t_{2}} J_{2}\left[\Delta \Sigma_{i j}\left(t_{1}, t_{2}\right)\right]\right\} .
$$

The limit for the Sines and the Crossland criteria will be:

$$
\begin{gathered}
\Delta \tau_{o c t, a}+B \cdot P_{m} \leq A, \\
\Delta \tau_{o c t, a}+B \cdot P_{\max } \leq A .
\end{gathered}
$$

\subsubsection{Dang Van Criterion}

Dang Van [8] postulates that the basic mechanism of fatigue cracks is the maximum shear of the most unfavourably positioned crystallographic plane. The material is considered homogeneous and isotropic at the macroscopic scale and is composed of monocrystals (or grains) with uncertain positions. The unfavourably positioned grains can be represented by an elastoplastic insertion immersed in an elastic matrix. The first cycles produce plastic slip at the grain level, and are rapidly stabilized: there is local elastic shakedown of the material. The fatigue criterion is expressed as a linear combination of the local shear stress in these grains and the concomitant hydrostatic pressure:

$$
\tau_{(n, t)}+\alpha \cdot P(t)=\beta
$$

The shear expression is obtained by using the Tresca criterion:

$$
\tau(t)=\max \left\{\operatorname{Tresca}\left[\sigma_{i j}(t)\right]\right\} .
$$

The model is represented in the plane $[\tau(t), P(t)]$; every couple $[\tau(t), P(t)]$ concerns a particular facet and is situated in the half-plane position $\tau(t)$. The constants $\alpha$ and $\beta$ are determined from two tests under simple loading. At least two Wöhler curves are to calculate their constants can also be used. In this case, the Wöhler curves used have a $50 \%$ probability of rupture. 


\subsubsection{Lu Criterion}

This criterion [9] is based on the amplitude of the octahedral shear stress $\left(\tau_{o c t}\right)$ or the maximum shear stress $\left(\tau_{\max }\right)$, and the maximum pressure $P_{\max }$. For different materials, $\tau_{o c t}$ or $\tau_{\max }$ is the critical plane. The basic criterion can therefore be expressed as follows:

$$
\tau_{\max , a} \quad \text { or } \quad \tau_{o c t, a}=f\left(A P_{m}, B P_{a l t}, C\right) .
$$

An example can be given using the following formula:

$$
\tau_{\max , a}+A\left(P_{m}\right)^{D}+B\left(P_{a l t}\right)^{E} \leq C,
$$

where $A, B, C, D$ and $E$ are material constants.

- If $\tau_{a}$ is taken in the maximum shear plane $\left(\tau_{\max , a}\right)$, then $D=E=1$ and $A=B$, which gives the Dang Van criterion.

- If $\tau_{a}$ is taken in the octahedral shear plane $\left(\tau_{\text {oct }, a}\right)$, when:

- $D=E=1, A=B$; we obtain the Crossland criterion,

- $D=E=1, B=0$; we obtain the Sines criterion,

- $D=E=1, A \neq B$; we obtain the Kakuno criterion [10].

This type of development can be continued to invent new criteria, but it only makes matters more complicated because more and more parameters have to be determined, since even with a Dang Van linear relationship, two Wöhler curves have to be determined to obtain at least the two points needed to construct the diagram. This criterion only becomes complex with particular loading and materials. As a result, it is the only fatigue criteria formula that can be used for a high number of cycles. However, the basic difference between this proposal and the other three criteria is obvious for non-proportional loading and in presence of stress concentration. In this case, the new criterion is proposed to make calculations at critical depths with stabilized states (elastic shakedown or plastic shakedown), and to take the combined out-of-phase stress effect into account using maximisation on all axes. For a combined torsion/bending out-of-phase stress, the Dang Van criterion can be written in the following form:

$$
\begin{gathered}
\frac{1}{2} \sqrt{\left(\sigma_{a} \sin (\omega t)\right)^{2}+4\left(\tau_{a} \sin (\omega t+\psi)\right)^{2}}= \\
=A+\frac{B}{3}\left(\sigma_{m}+\sigma_{a} \sin (\omega t)\right) .
\end{gathered}
$$

Since this formula does not take the results during dephasing stress into account with sufficient precision [7], we suggest modifying it as follows: shear amplitude according to Tresca or Mises ( $\tau_{a}$, maximized on all the component axes and for all the terms of the loading sequence) $=$ $\mathrm{A}+\mathrm{B} \cdot P_{\max }$. For a combined torsion/bending outof-phase stress using the Tresca criterion, this gives:

$$
\begin{gathered}
\frac{1}{2} \sqrt{\left(\sigma_{a, \max (t)}\right)^{2}+4 k\left(\tau_{a, \max (t)}\right)^{2}}= \\
=A+\frac{B}{3}\left(\sigma_{m}+\sigma_{a, \max (t)}\right),
\end{gathered}
$$

$k$ is a so-called phase lag-sensitive coefficient. It depends on the nature of the material, the frequency, level and type of the stress. If there is no stress dephasing, then $k=1$. The physical signifiance of this formula is as follows: when there is dephasing stress, the time needed by the material to react depends on the nature of the material and the loading level. The material remembers, as it were, the maximum previous stress along another stress axis. Therefore, the stress level to be taken into account is not the maximum stress along one axis and the instantaneous stress along the other axes, but the maximum stress $\tau_{a 1, \max }, \tau_{a 2, \max }$ and $\tau_{a 3 \text {, max }}$ along all the axes. Where, for $i=1,3$ :

$$
\tau_{a i, \max \left(t_{1}, t_{2}\right)}=\max _{t_{1}}\left\{\max _{t_{2}}\left[\tau_{a i\left(t_{1}, t_{2}\right)}\right]\right\} .
$$

When the stress frequency is high, the time needed for the memory effect to occur is even lower still. When there is stress dephasing and if $k$ is between 0 and 1 , the errors are relatively small. Using $k=1$ for high cycles fatigue limit when the loading frequency is higher than $15 \mathrm{~Hz}$, a maximum underestimation of the fatigue resistance corresponding to $10 \%$ is observed. Thus, the general formula depends on the maximum stress along all the axes, and coefficient $k$ : 


$$
\tau_{\max }=f\left(\tau_{a i, \max \left(t_{1}, t_{2}\right)}, k\right)
$$

\subsection{Fatigue Life Prediction}

We have developed a global approach to calculate the fatigue life of 3D structures [11]. The numerical results obtained by using a finite element method, together with the tests results, were introduced into a main program (Fig. 4). The results are shown by graphic representation of the fatigue life of the structure. The mechanical characteristics of the selected material, together with the fatigue data in the form of Wöhler curves, are recovered. In our case, the Wöhler curves we used have a $50 \%$ probability of rupture. For a criterion with two constants, such as that of Crossland, Sines or Dang Van, at least two Wöhler curves are needed to calculate their constants. In the case of simple or combined in-phase loadings, the Sines, Crossland or Dang Van criterion can be used. When there is combined out-of-phase loading, the $\mathrm{Lu}$ criterion is added to the list of possible criteria. Once the two constants have been calculated for the fatigue criterion chosen, the corresponding line on the diagram for this criterion can be plotted. This line will correspond to a particular number of cycles.

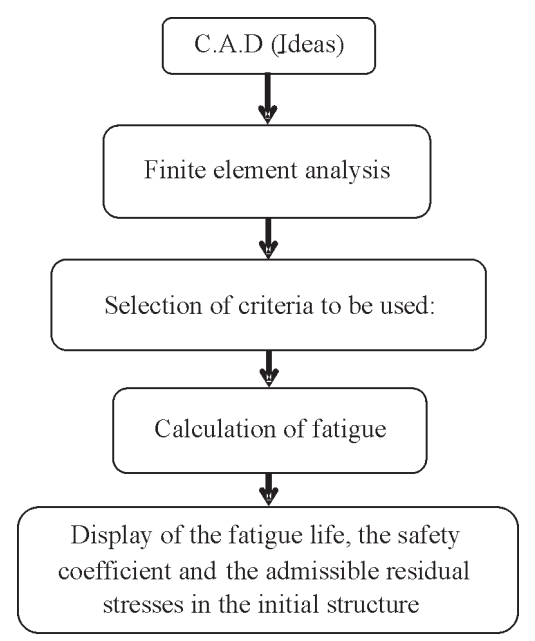

Fig. 4. Fatigue life calculation

Likewise, several lines can be plotted on the criterion diagram (each line corresponds to a specific number of cycles), the coefficients of which are calculated using the Wöhler curves (Fig.
5). This method is normally valid in the field of endurance, but we have extended the use of the fatigue criterion to include other field. An example of plotting of life contour is given for a notched specimen subjected to bending. The structure studied is modelled with CAD software to provide data in standardized form. The boundary conditions and the loading are assigned to the structure. With finite element software such as Abaqus or I-DEAS, an elastic or elastoplastic analysis can be carried out. The results of calculations based on the finite element method, in the form of stress tensors, are recovered and integrated into the fatigue life calculation module. As a result, the fatigue life in the diagram for the criterion used can be mapped. This means that, at any point on the criterion diagram, there is the corresponding fatigue life.

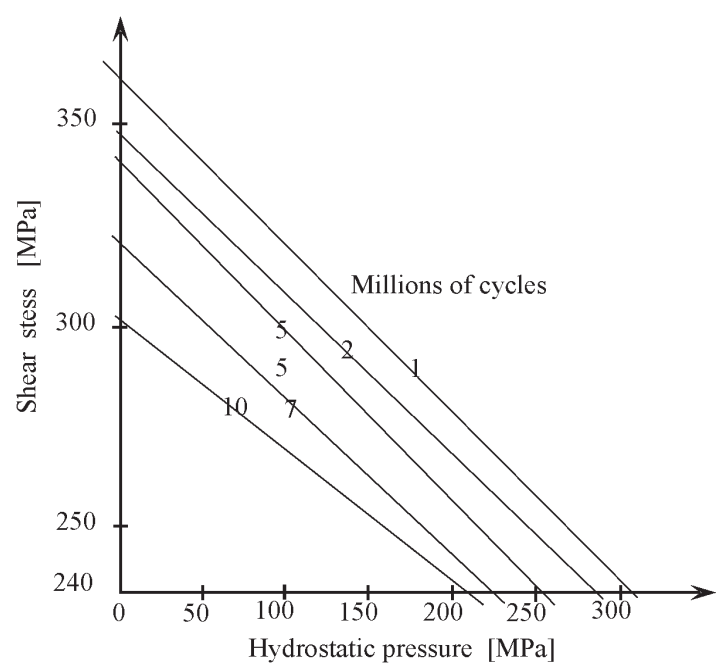

Fig. 5. Map of fatigue life in $(\tau, P)$ diagram

This approach is applied to simple loading (alternate tension, alternate bending, etc. or combined loading (bending-tension, torsiontension, etc.) whether it is in-phase or out-ofphase. If it is out-of-phase, the method proposed by $\mathrm{Lu}$ is used to prove the applicability of the method and to take the out-of-phase effect into account.

An example of application of this method relates to two different notched specimens subjected to the same loading. The specimens have stress concentration coefficients corresponding to: $k_{t 1}=1.3$, and $k_{t 2}=1.7$. Tests were carried out 
on FGS cast iron. The mechanical properties for this material are tensile strength of $750 \mathrm{MPa}$, a Young's modulus of $162.2 \mathrm{GPa}$ and a yield stress of $416 \mathrm{MPa}$. The bending fatigue strength is 294 $\operatorname{MPa}(R=-1)$, and the torsion fatigue strength is $218 \mathrm{MPa}(R=-1)$. The fatigue life is calculated for the two specimens using the Crossland fatigue criterion. The computational results are illustrated for the initial specimen in the form of cartography (Fig. 6).
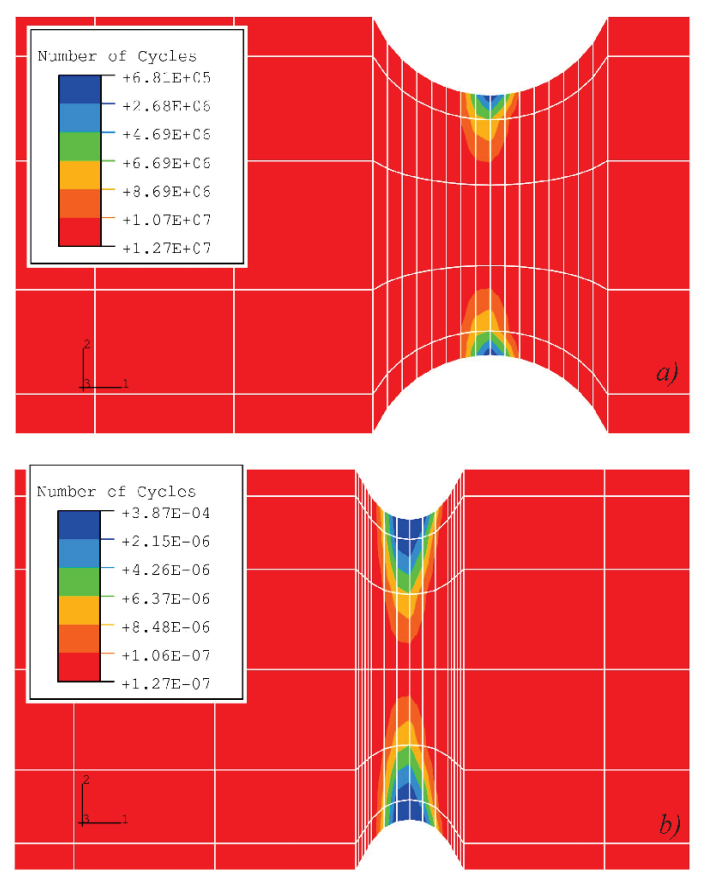

Fig. 6. Prediction of fatigue life for two different specimens; a) a coefficient of stress concentration $\left.k_{t 1}=1.3, b\right)$ a coefficient of stress concentration

$$
k_{t 2}=1.7
$$

These results show a clear difference in fatigue life prediction when the notch shape changes. Specimen with a coefficient of stress concentration equal to $k_{t 1}=1.3$ has a longer fatigue life than those coefficient of stress concentration is equal to $k_{t 2}=1.7$. An example is given of the predicted fatigue life in the case of a combined bending/torsion out-of-phase (phase angle $90^{\circ}$ ). In this case, the best fatigue life prediction results were obtained with a dephasing-sensitive coefficient $k=0.13$ for Lu criterion. This criterion can therefore, be applied successfully in the case of combined out-of-phase loading. The results obtained for the different criteria using the Wöhler curves are compared with the experimental results (Fig. 7).

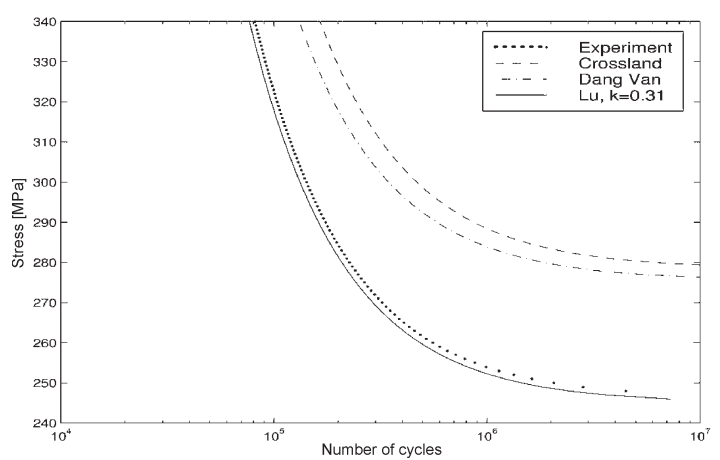

Fig. 7. Prediction of Wöhler curves

\subsection{Prediction of Admissible Residual Stress and the Safety Coefficient}

In this approach [11], a calculation method for predicting the admissible residual stress and the safety coefficient for a particular fatigue life has been developed. It is thus possible to use this tool during the design phase to determine whether or not residual compressive stress should be introduced. The computer code also indicates the level of the residual stress and the zone in which it should be introduced.

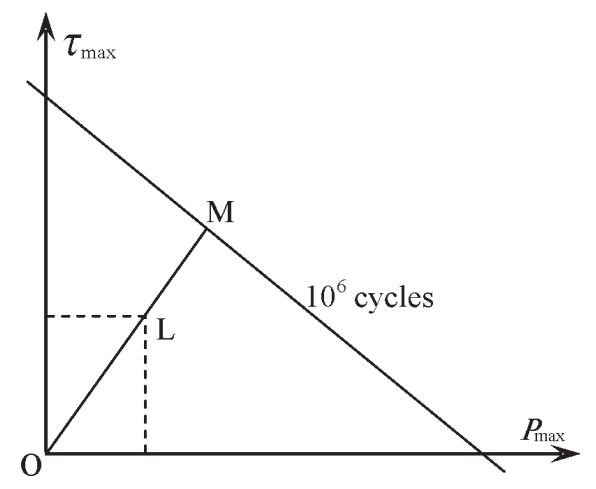

Fig. 8. Calculation of safety coefficient in $(\tau, P)$ diagram

The purpose of the first method is to predict the safety coefficients of a structure for a particular fatigue life in order to know the performance to 
be improved. The same notched specimens as for he prediction of the fatigue life were used. In this part, only the Crossland criteria will be used. The general principle of the calculation of the safety coefficient is illustrated in Fig. 8 with any fatigue criterion (Crossland, Sines, Haigh or Goodman). The simulation was carried out on FGS cast iron in bending. The targeted fatigue life for our specimen was $10^{6}$ cycles. For this desired fatigue life, the representative point " $\mathrm{L}$ " of a tensile load is placed in the diagram of the Crossland criterion. We then calculate the value of the safety coefficient which is given by the following Eq.:

$$
s=O M / O L
$$

Using the Wöhler curves of FGS cast iron, admissible residual stress are predicted for a particular fatigue life of $10^{6}$ cycles represented by the line drawn in the diagram of the Crossland criterion (Fig. 9).

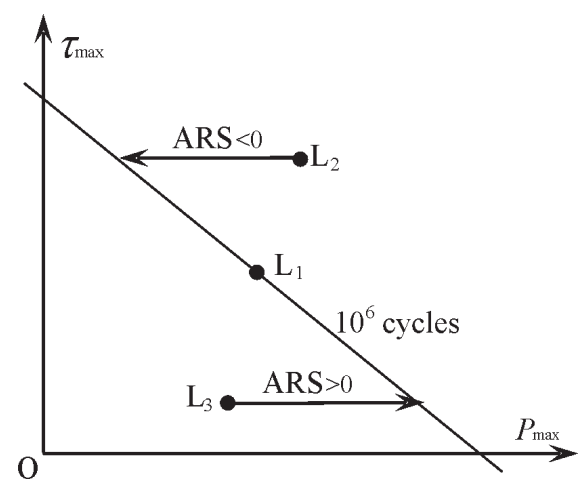

Fig. 9. Calculation of admissible residual stress $(A R S)$ in $(\tau, P)$ diagram

For a load equivalent to the amplitude $L_{1}$ or $L_{3}$, the fatigue life is equal or greater than $10^{6}$ cycles. It is not necessary, therefore, to introduce residual stresses in the structure.

When the load $L_{2}$ is applied, it induces a short fatigue life. To achieve a fatigue life of $10^{6}$ cycles, negative admissible residual stresses (ARS) in the structure can be introduced. The idea is to provide these constraints and also the different surface treatments applied to achieve the desired fatigue life.

In case applying the load $L_{3}$, its fatigue life is greater than $10^{6}$ cycles. Then residual stresses will not need to be introduced, and consequently, no surface treatment.

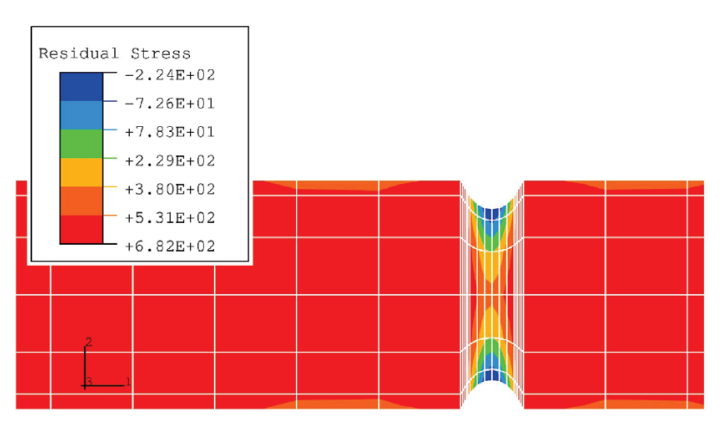

Fig. 10. Prediction of admissible residual stress for a targeted lifetime of $10^{6}$ cycles [MPa]

By mapping the safety coefficients for a particular fatigue life, the areas with the highest risk which must be modified accordingly during the design and manufacturing stage can also be predicted. It has been observed (Fig. 10) that fatigue life is minimal in zones with high stress concentration. In these same zones, residual compressive stress must be introduced in order to achieve the required fatigue resistance of the structure. The predicted safety coefficients in bending needed for the structure to resist fatigue up to $10^{6}$ cycles (Fig. 11) was also mapped.

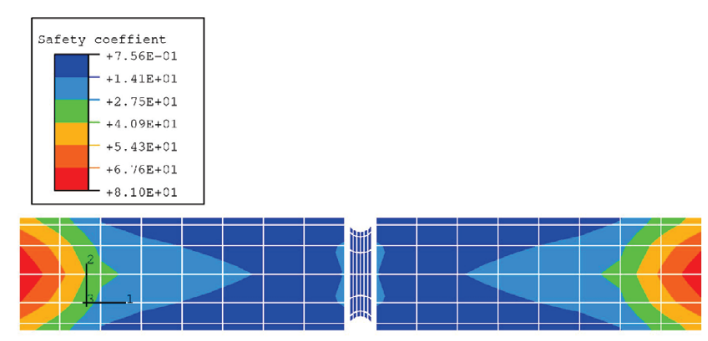

Fig. 11. Prediction of safety coefficient for a targeted life of $10^{6}$ cycles

\section{CONCLUSION}

As part of a global approach to integrated mechanical design, a tool for calculating fatigue life, which is compatible with industrial software such as Abaqus and I-DEAS, has been developed. The tool allows no proportional multiaxial loading 
and residual stress to be taken into account. By combining it with other tools developed in our laboratory, components and mechanical systems can be optimized. For a given lifetime of a particular material and loading, modelling can be used to indicate whether prestressed is necessary. The areas to be reinforced together with the prestressed conditions required can also be mapped. In this way, a genuine prestressed engineering strategy can be developed. Residual stress will no longer be considered the result of a manufacturing process, but a modifiable parameter. It can thus be optimised according to mechanical loading.

\section{ACKNOWLEDGEMENTS}

The authors would like to thank the Ministry of Research and Technology for the financial support thus enabling this research.

\section{REFERENCES}

[1] Lafon, P. (1994). Optimal design of mechanical system: optimization of variables mixed. $\mathrm{PhD}$, INSA Toulouse. (in French)

[2] Lu, J. (ed.) (1996). Handbook of measurement of residual stresses. The Fairmont Press-Prentice Hall.

[3] Lu, J., Flavenot, J.F., Turbat, A. (1988). Prediction of residual stress relaxation during fatigue, Mechanical relaxation of residual stresses. ASTM STP993, p. 75-90.

[4] Lu, J., Flavenot, J.F., Turbat, A. (1988). Residual stress relaxation under cyclic loading: Influence of the mechanical properties and prediction by calculation,
Memory and scientific study, p. 615-626. (in French)

[5] Rouhaud, E., Milley, A., Lu, J. (1997). Introduction of residual stress fields in finite element three-dimensional structures. $5_{\text {th }}$ Int. Conf. on Residual Stresses, ICRS, Linkoping

[6] Sines, G. (1981). Fatigue criteria under combined stress or strain. Transactions ASME, J. Eng. Mat. and Tech., vol. 13, p. 82-90.

[7] Crossland, B. (1956). Effect of large hydrostatic pressure on the torsional fatigue strength of an alloy steel. Int. Conf. on fatigue of metals, IME/ASME, p. 138-149.

[8] Dang Van, K., Cailletaud, G., Flavenot, J. F., Douaron, Lieurade, H.P. (1984). Fatigue initiation criterion to large numbers of cycles under multiaxial stress. J. Int. de Printemps: Amorçage des fissures sous sollicitations complexes, French Society of Metallurgy, p. 301-337.

[9] Lu, J., Flavenot, J.F., Diboine, A., Lasserre, S., Froustey, C., Bennebach C., Palin-Luc, T. (1996). Development of a general multiaxial fatigue criterion for high cycles of fatigue behaviour prediction. Multiaxial fatigue and design, Pineau, A., Cailletaud, G., Lindley, T. C. (eds.), The European Structural Integrity Society, ESIS 21, Mechanical Engineering Publications, p. 477-487.

[10] Kakuno, H., Kawada, Y. (1979). A new criterion of fatigue strength of a round bar subjected to combined static and repeated bending and torsion. Fatigue of Eng. Mat. and Structures, vol. 2, p. 229-236.

[11] Akrache, R. (1998). Prediction of fatigue life of $3 D$ structures by the finite element method. PhD, UTT de Troyes. (in French) 